\title{
Some New Results in the Group-theoretical Description of the Radiation Transfer
}

\author{
A. Nikoghossian* \\ NAS RA V.Ambartsumian Byurakan Astrophysical Observatory, Byurakan 0213, Aragatsotn Province, Armenia
}

\begin{abstract}
The paper presents some new results of investigation developing the approach aimed at applying the group theory methods to radiation transfer problems. It consists of two separate parts. In the first part we derive new properties of supersymmetry of fundamental supermatrices - representations of composition and translation groups. It is shown that these supermatrices, which determine the layers adding to the two opposite boundaries of inhomogeneous medium, are connected with each other by the procedure of parity transposition. It is also demonstrated that, by analogy with the common second order matrices, the considered supermatrices can be factorized yielding the product of triangular supermatrices. The second part generalizes and applies the concept of composition groups to the case of media with spherical symmetry.
\end{abstract}

\section{Inroduction}

The bases of application of the group theory methods in the problems of radiative transfer are given in a series of author's papers (Nikoghossian, 2014, 2019). The importance of such application is determined by at least two main factors. First of all, it must be noted its value at revealing symmetry and supersymmetry properties in problems of the transfer theory and in establishing the close connection between various problems of astrophysical importance, on one hand, and different classical methods of solution, on the another hand. The symmetry properties, in turn, has enabled to propose the Lagrange-Hamiltonian approach (Nikoghossian, 1999, 2013) and to derive conservation laws in many classical problems. Thanks to this approach, many of the basic physical properties of the problem under study can be established before the problem is solved. Finally, the found representations of composition and translation groups offer new simpler schemes of both analytical and numerical solution of realistic and then rather complex radiation transfer problems,

In this paper, we will first present some new relations between the basic supermatrices introduced by the author in connection with the symmetry properties of the problem of radiation transfer in the medium of finite optical thickness. Further on, we generalize some of the results obtained for the plane-parallel media to the case of media with spherical symmetry.

\section{The symmetry properties of basic super-matrices.}

In the mentioned fundamental works (Nikoghossian, 2011, 2014) we have obtained the representations of composition groups in the form of second order supermatrices, which establish the adding law for global optical properties (reflection and transmittance coefficients) of scattering and absorbing media when being they combined. It was also shown that these representations also describe the transition from one optical depth to the another, which, in determining the field of radiation inside the medium, can be considered as a law of translation between different depths. These laws obtained by applying the group theory, in fact, can be considered as a further development Ambartsumian's idea, which is the basis of his method of layers addition (Ambartsumian, 1944, Ambartsumyan, 1960). They

*nikoghoss@yahoo.com 
cover a wide range of various problems of radiation transfer theory, involving important problems of theoretical astrophysics.

In this section, we show how the forms of representations of composition groups are related with each other in the most general case of inhomogeneous medium. We remind that, when the inhomogeneous medium is illuminated from the side of one of its boundaries, the addition of global optical characteristics is carried out with the help of supermatrices as follows

$$
\tilde{\mathbf{A}}=\left(\begin{array}{cc}
\mathbf{P} & -\overline{\mathbf{S}} \\
\mathbf{S} & \mathbf{M}
\end{array}\right), \quad \tilde{\mathbf{B}}=\left(\begin{array}{cc}
\mathbf{M} & -\mathbf{S} \\
\overline{\mathbf{S}} & \mathbf{P} .
\end{array}\right)
$$

where we have used the notations introduced in Nikoghossian (2014): $\mathbf{P}=\mathbf{Q}^{-\mathbf{1}}, \mathbf{S}=\mathbf{R} \mathbf{P}, \overline{\mathbf{S}}=$ $\mathbf{P} \overline{\mathbf{R}}, \mathbf{M}=\mathbf{Q}^{*}-\mathbf{S} \overline{\mathbf{R}}$. Here $\mathbf{R}$ and $\overline{\mathbf{R}}$ are the reflectance of the medium of two mutually opposite sides and $\mathbf{Q}, \mathbf{Q}^{*}$ are corresponding transmission coefficients. As usual, the transposed matrices are supplied by asterisks. The properties of these supermatrices were studied in the mentioned papers and were particularly shown their invertibility given by

$$
\tilde{\mathbf{A}}^{-1}=\left(\begin{array}{cc}
\mathbf{M}^{*} & \overline{\mathbf{S}}^{*} \\
-\mathbf{S}^{*} & \mathbf{P}^{*}
\end{array}\right), \quad \tilde{\mathbf{B}}^{-1}=\left(\begin{array}{cc}
\mathbf{P}^{*} & \mathbf{S}^{*} \\
-\overline{\mathbf{S}}^{*} & \mathbf{M}^{*}
\end{array}\right) .
$$

Similarly, with the same designations and the same direction of reference, if the medium is illuminated from the opposite side, the supermatrixes of the compositions group are

$$
\tilde{\mathbf{C}}=\left(\begin{array}{cc}
\mathbf{P}^{*} & -\mathbf{S}^{*} \\
\overline{\mathbf{S}}^{*} & \mathbf{M}^{*}
\end{array}\right), \quad \tilde{\mathbf{D}}=\left(\begin{array}{cc}
\mathbf{M}^{*} & -\overline{\mathbf{S}}^{*} \\
\mathbf{S}^{*} & \mathbf{P}^{*}
\end{array}\right) .
$$

which are also non-singular

$$
\tilde{\mathbf{C}}^{-1}=\left(\begin{array}{cc}
\mathbf{M} & \mathbf{S} \\
-\overline{\mathbf{S}} & \mathbf{P}
\end{array}\right), \quad \tilde{\mathbf{D}}^{-1}=\left(\begin{array}{cc}
\mathbf{P} & \overline{\mathbf{S}} \\
-\mathbf{S} & \mathbf{M}
\end{array}\right)
$$

These supermatrices are fundamental in the developed theory since they establish the laws of transformation of global optical characteristics in composing scattering and absorbing media. All of them have a number of common properties, of which, except invertibility, we note the equality to unity of their superdeterminants (Nikoghossian, 2014, 2019). Being the supermatrices of the second order, they, by analogy with usual matrices, can be represented in the form of the product of two triangular supermatrices. So, for example, in the case of the supermatrix $\mathbf{A}$, one can write an easily verifiable equality

$$
\tilde{\mathbf{A}}=\left(\begin{array}{cc}
\mathbf{I} & \mathbf{0} \\
\mathbf{R} & \mathbf{I}
\end{array}\right)\left(\begin{array}{cc}
\mathbf{P} & -\overline{\mathbf{S}} \\
\mathbf{0} & \mathbf{Q}^{*}
\end{array}\right)
$$

where $\mathbf{I}$ is the unit matrix. The supermatrices we have introduced, along with the inverse ones describe the same composition of the medium illuminated from two mutually opposite sides. This obviously means that there must be a relationship between these group representations with use of the concepts of supertransposition and transposition of parity

$$
\begin{array}{ll}
\left(\tilde{\mathbf{A}}^{s t}\right)^{s t}=\tilde{\mathbf{D}}^{-1}, \quad & \left(\tilde{\mathbf{B}}^{s t}\right)^{s t}=\tilde{\mathbf{C}}^{-1}, \\
\left(\tilde{\mathbf{D}}^{s t}\right)^{s t}=\tilde{\mathbf{A}}^{-1}, & \left(\tilde{\mathbf{C}}^{s t}\right)^{s t}=\tilde{\mathbf{B}}^{-1},
\end{array}
$$

where the upper index st means supertransposition. There are more simple relations which use the concept of the parity transposition marked by the upper index $\pi$

$$
\tilde{\mathbf{A}}^{\pi}=\tilde{\mathbf{C}}^{-1}, \quad \tilde{\mathbf{B}}^{\pi}=\tilde{\mathbf{D}}^{-1}, \quad \tilde{\mathbf{C}}^{\pi}=\tilde{\mathbf{A}}^{-1}, \quad \tilde{\mathbf{D}}^{\pi}=\tilde{\mathbf{B}}^{-1} .
$$

Finally, the results obtained for representation of composition groups for two mutually opposite directions in the general case of inhomogeneous media can be presented as follows

$$
\left(\begin{array}{cc}
\mathbf{A} & \mathbf{0} \\
\mathbf{0} & \mathbf{B}
\end{array}\right)\left(\begin{array}{cc}
\mathbf{C}^{\pi} & \mathbf{0} \\
\mathbf{0} & \mathbf{D}^{\pi}
\end{array}\right)=\left(\begin{array}{cc}
\mathbf{C} & \mathbf{0} \\
\mathbf{0} & \mathbf{D}
\end{array}\right)\left(\begin{array}{cc}
\mathbf{A}^{\pi} & \mathbf{0} \\
\mathbf{0} & \mathbf{B}^{\pi}
\end{array}\right)=\left(\begin{array}{cc}
\mathbf{I} & \mathbf{0} \\
\mathbf{0} & \mathbf{I}
\end{array}\right)
$$




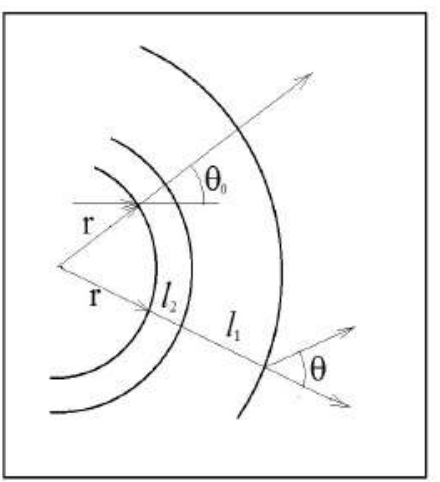

Figure 1. The schematic picture of radiating one-dimensional turbulent atmosphere

\section{Representations of composition groups for media with spherical symmetry}

We now show how the approach elaborated for plane-parallel media in Nikoghossian $(2014,2019)$ can easily be generalized to the case of media with spherical symmetry. Such generalization is of great aetrophysical interest, especially when we deal with extended atmospheres of stars. From mathematical point of view, the study of media with spherical symmetry generally is more complex because of appearance of additional parameters due to the curvature and its effects. Therefore, any progress that contributes to the solution of the problems of multiple light scattering in the media with spherical geometry is of great importance.

Let us start with considering the problem of radiation transfer through two spherical, generally inhomogeneous layers, which are illuminated from the inner spherical region with radius $r$. Each layer will be specified by its internal radius and optical thickness $\tau_{0}$ with corresponding geometric thicknesses $\ell$ calculated along the normal. The incident radiation is supposed to be isotropic. The angles of incidence of radiation on the medium and its exit are correspondingly designated through $\vartheta_{0}$ and $\vartheta$ (Fig. 1). We also suppose that the inner spherical core does not take part in the multiple scattering process, i.e., the photons reflected from the shells do not turn back to it from the core. Evidently, such assumption may be regarded as realistic for sufficiently distant shells. On the other hand, this assumption is crucial from the point of view of application of the developed theory to this simplified model case.

We are interested in global optical characteristics of the composite atmosphere, provided that these characteristics for constituent layers are known. In this formulation of the problem, it is possible to apply the procedure of combining the scattering spherical layers, which is in many respects similar to the composition of planar media considered in Nikoghossian $(2014,2019)$. Here also, as a binary operation we can choose the result of two successive transformations that present addition of new layers. Then it is easy to check that the group properties of the composition procedure are satisfied. The composition groups for spherical layers are characterized by their non-commutativity, which in contrast to plain-parallel media, occurs even if the added media are homogeneous. In general, the treated group of compositions is two-parametric, where as parameters can be chosen internal radius and optical or geometric thickness of the layer.

Following the approach suggested in Nikoghossian (2014), the reflection and transmission coefficients of the layer in the discrete space can be represented in the form of matrices, denoted as $\mathbf{R}\left(\mathbf{r}, \tau_{\mathbf{0}}\right)$ and $\mathbf{Q}\left(\mathbf{r}, \tau_{\mathbf{0}}\right)$. Now the elements of fundamental matrices $\mathbf{P}, \mathbf{S}, \overline{\mathbf{S}}, \mathbf{M}$ in the representation of the composition groups associated with the probability of reflection and transmission depend also on the thickness of the layer and its curvature. The process of radiation diffusion depending on the frequency will not be considered here.

Fig,1 schematically shows the composition of two spherical layers, with the layers numbering chosen in such a way that it together with the direction of the incident radiation coincide with those in the 
model used in Nikoghossian (2014). This will make it possible to preserve both the reasoning and resulting relations obtained for plane-parallel media. The reasoning used in the mentioned paper to obtain the representations of a group of compositions also remain valid. Referring the reader to this work for the course of argumentation, we point out that

$$
\mathrm{Q}_{1 \cup 2}=\mathrm{Q}_{1} \mathrm{TQ}_{2}
$$

is also true for spherical media in our case. The reasoning also remains in force, in deriving the first important relation, which connects the values of $\mathbf{P}, \mathbf{S}, \overline{\mathbf{S}}$ of the two media

$$
\mathbf{P}_{1 \cup 2}=\mathbf{P}_{2} \mathbf{P}_{1}-\overline{\mathbf{S}}_{2} \mathbf{S}_{1}
$$

In the expanded form the equation (10) for our case will be rewritten as

$$
\mathbf{P}\left(\mathbf{r}, \ell_{\mathbf{1}}+\ell_{\mathbf{2}}\right)=\mathbf{P}\left(\mathbf{r}, \ell_{\mathbf{2}}\right) \mathbf{P}\left(\mathbf{r}+\ell_{\mathbf{2}}, \ell_{\mathbf{1}}\right)-\overline{\mathbf{S}}\left(\mathbf{r}, \ell_{\mathbf{2}}\right) \mathbf{S}\left(\mathbf{r}+\ell_{\mathbf{2}}, \ell_{\mathbf{1}}\right)
$$

Analogously, we have

$$
\mathbf{S}\left(\mathbf{r}, \ell_{1}+\ell_{\mathbf{2}}\right)=\mathbf{S}\left(\mathbf{r}, \ell_{\mathbf{2}}\right) \mathbf{P}\left(\mathbf{r}+\ell_{\mathbf{2}}, \ell_{\mathbf{1}}\right)+\mathbf{M}\left(\mathbf{r}, \ell_{\mathbf{2}}\right) \mathbf{S}\left(\mathbf{r}+\ell_{\mathbf{2}}, \ell_{\mathbf{1}}\right)
$$

Thus, in this formulation of the problem, the transformation of the basic matrices is carried out by means of the supermatrix which is the natural generalization of that of the same name in the case of the planar geometry

$$
\tilde{\mathbf{Y}}\left(\mathbf{r}, \ell_{\mathbf{1}}+\ell_{\mathbf{2}}\right)=\tilde{\mathbf{A}}\left(\mathbf{r}, \ell_{\mathbf{2}}\right) \tilde{\mathbf{Y}}\left(\mathbf{r}+\ell_{\mathbf{2}}, \mathbf{l}_{\mathbf{1}}\right)
$$

where, as before in $[1]$

$$
\tilde{\mathbf{Y}}=\left(\begin{array}{c}
\mathbf{P} \\
\mathbf{S}
\end{array}\right) \quad \tilde{\mathbf{A}}=\left(\begin{array}{cc}
\mathbf{P} & -\overline{\mathbf{S}} \\
\mathbf{S} & \mathbf{M}
\end{array}\right)
$$

For simplicity, we will conduct further reasoning for homogeneous layers, i.e. we assume that the physical parameters determining the elementary act of scattering does not vary throughout of the medium. Then, for the infinitesimal operator of the two-parametric group under consideration, we have

$$
\tilde{\Xi}(r, 0)=\lim _{\Delta \ell \rightarrow 0} \frac{\tilde{\mathbf{A}}(r, \Delta \ell)-\mathbf{E}}{\Delta \ell}=\left(\begin{array}{cc}
\mathbf{m} & -\mathbf{n} \\
\mathbf{n} & -\mathbf{m}
\end{array}\right),
$$

where we have introduced notations

$$
\mathbf{m}=\frac{\mathbf{1}}{\xi}\left[\mathbf{I}-\frac{\lambda}{\mathbf{2}} \mathbf{x}(\gamma)\right], \quad \mathbf{n}=\frac{\lambda}{\mathbf{2} \xi} \mathbf{x}(\gamma), \quad \tilde{\mathbf{E}}=\left(\begin{array}{cc}
\mathbf{I} & 0 \\
0 & \mathbf{I},
\end{array}\right)
$$

and $\xi=\cos \theta_{0}, \eta=\cos \theta, \mathbf{x}(\gamma)$ is the diffusion indicatrix dependent on the scattering angle $\gamma$, and $\lambda$ is the coefficient of re-radiation of quantum in the elemetary act of scattering.

With use of (16) the relations (12), (13) can be written in the differential form. To this end we replace $\ell_{2}$ by infinitesimal thickness $\Delta \ell$ and $\ell_{1}$ by $\ell$. Then passing to the limit when $\Delta \ell \rightarrow 0$, we find

$$
\begin{aligned}
& \frac{\partial \mathbf{P}}{\partial \ell}-\frac{\partial \mathbf{P}}{\partial r}=\mathbf{m} \mathbf{P}(r, \ell)-\mathbf{n S}(r, \ell) \\
& \frac{\partial \mathbf{S}}{\partial \ell}-\frac{\partial \mathbf{S}}{\partial r}=\mathbf{n P}(r, \ell)-\mathbf{m S}(r, \ell)
\end{aligned}
$$

with the initial conditions $\mathbf{P}(\mathbf{r}, \mathbf{0})=\mathbf{I} / \xi, \mathbf{S}(\mathbf{r}, \mathbf{0})=\mathbf{0}$. Thus, we are led to a set of two partial differential linear equations which with the proper parametrisation may be reduced to a set of ordinary differential equations for the functions $\overline{\mathbf{P}}(t) \equiv \mathbf{P}(r, t+\ell), \overline{\mathbf{S}}(t) \equiv \mathbf{S}(r, t+\ell)$. This kind of two-parametric equations we have already obtained in considering the model non-stationary transfer problem as well as the problem of multiple scattering in the turbulent media (see Nikoghossian (2019)). There are standard classical methods used equation of characteristics (see e.g., Courant \& Hilbert (1962), 
Rojdenstvenski \& Yanenko (1968)upon which we do not dwell here. In the case of the model problem treated in the present paper, the curvature of the medium mathematically affects only the form of the resulted equation, meanwhile the solution of the problem we are led is equivalent to that for the plain-parallel atmosphere of geometrical thickness $\ell$ (Nikoghossian, 2012). The approach we adopt in the paper can be applied to the more general problem of astrophysical interest when the target value is the observed intensity, i.e., that along the line of sight. This leads to appearance of the new parameter denoting the angle between direction of the photons exit and the line of sight (see e.g., Mihalas (1978), Sobolev (1963). This three-parametric problem will be serve as a subject of one of our future works.

\section{References}

Ambartsumian V. A., 1944, Izv. Akad. Nauk ArmSSR, N 1, 2

Ambartsumyan V. A., 1960, Scientific Works, V. I, Yerevan,Izd. Acad. Nauk ArmSSR

Courant R., Hilbert D., 1962, Methods of Mathematical Physics, V. II

Mihalas D., 1978, Stellar atmospheres, San-Francisco, Freeman

Nikoghossian A. G., 1999, JQSRT, 61, 345

Nikoghossian A. G., 2011, Astrophysics, 54, 553

Nikoghossian A. G., 2012, Astrophysics, 55, 261

Nikoghossian A. G., 2013, Light Scat. Reviews, 55, 377

Nikoghossian A. G., 2014, Astrophysics, 57, 272

Nikoghossian A. G., 2019, Astrophysics, 62, 92

Nikoghossian A. G., 2014a, Astrophysics, 57, 375

Rojdenstvenski B. L., Yanenko N. N., 1968, Systems of Quasi-linear Equations and Their Application to Gas Dynamics, M., Nauka,

Sobolev V. V., 1963, A Treatise on Radiative Transfer, Princeton: van Nostrand., 